\title{
TAUBERIAN THEOREMS AND TAUBERIAN CONDITIONS
}

BY

\section{G. G. LORENTZ}

1. Introduction. The chief aim of this paper is an investigation of relations among Tauberian theorems. In $\$ 2$ we compare " high indices theorems" or "gap theorems" with "order" Tauberian theorems containing a condition of the form $u_{n}=o\left(c_{n}\right)$ or $u_{n}=O\left(c_{n}\right)$. Especially for the methods of Abel and Cesàro we shall look for necessary and sufficient conditions on the numbers $c_{n}$, for which these theorems are valid.

2. Relations among Tauberian theorems. Let $A$ be a Toeplitz-Silverman method of summation, given by the transformation

$$
\sigma_{m}=a_{m 1} s_{1}+a_{m 2} s_{2}+\cdots+a_{m n} s_{n}+\cdots
$$

of the sequence $s_{n}$ into the sequence $\sigma_{m}$. We shall call the sequence $s_{n}$ and the series $\sum u_{n}$ with the partial sums $s_{m} A$-summable to the value $s$, if $\lim \sigma_{m}=s$. We assume that the conditions of regularity for the method $A$ are fulfilled. Then from $s_{n} \rightarrow s$ we have $\sigma_{m} \rightarrow s$. A Tauberian theorem for the method $A$ is a proposition in which, conversely, $s_{n} \rightarrow s$ is deduced from $\sigma_{m} \rightarrow s$ and an additional condition on the series $\sum u_{n}$ or the sequences $s_{n}$. This latter condition is called a Tauberian condition for the method $A$.

Let $n_{k}$ be a sequence $n_{1}<n_{2}<\ldots$ of positive integers. The following propositions are called high indices or gap theorems for the method $A$.

$\left(\mathrm{H}_{1}\right)$. If a series $\sum u_{n}$ is $A$-summable and satisfies the gap condition

$$
u_{n}=0,
$$$$
n \neq n_{1}, n_{2}, \cdots,
$$

then the series is convergent.

$\left(\mathrm{H}_{2}\right)$. If the A-transform $\sigma_{m}$ of the sequence $s_{n}=\sum_{k-1}^{n} u_{k}$ is bounded and if the series $\sum u_{n}$ satisfies (2), then $s_{n}$ is also bounded.

Let $c_{n}$ be any sequence of numbers $0 \leqq c_{n} \leqq+\infty$. In analogy to the above propositions, we state the following Tauberian theorems containing an estimate of $u_{n}$.

$\left(\mathrm{T}_{1}\right)$. If a series $\sum u_{n}$ is $A$-summable and satisfies the order condition

$$
u_{n}=o\left(c_{n}\right)
$$

then the series is convergent.

$\left(\mathrm{T}_{2}\right)$. If $\sigma_{n}$ is bounded and

$$
u_{n}=O\left(c_{n}\right)
$$

then $s_{n}$ is also bounded.

Presented to the Society, October 25, 1947; received by the editors February 18, 1947. 
Note that (3) and (4) impose no limitation for those $u_{n}$ for which $c_{n}=+\infty$. Hence it may happen that a high indices theorem is contained in an order Tauberian theorem. See Theorem 3 which, for the Abel method, contains as special cases the Tauberian theorem with the condition $u_{n}=O(1 / n)$ and the Hardy-Littlewood high indices theorem.

We now prove the following theorem.

THEOREM 1. If for the method $A$ the high indices theorem $\left(\mathrm{H}_{1}\right)$ is valid, and if further

$$
\sum_{n_{k}<n<n_{k+1}} c_{n}=O(1)
$$

then $\left(T_{1}\right)$ is also valid.

Proof. Suppose that, for an $A$-summable series $\sum u_{n}$, the condition (3) is fulfilled. Let $s_{n}^{\prime}=0$ when $n<n_{1}$ and $s_{n}^{\prime}=s_{n_{k}}$ when $n_{k} \leqq n<n_{k+1}, k=1,2, \cdots$. Then by means of the estimates (3) and (5) we get $s_{n}-s_{n}^{\prime} \rightarrow 0$. From the regularity of the method $A$ we now have for the $A$-transform $\sigma_{m}{ }^{\prime}$ of the sequence $s_{n}^{\prime}$

$$
\sigma_{m}-\sigma_{m}^{\prime}=\sum_{n=1}^{\infty} a_{m n}\left(s_{n}-s_{n}^{\prime}\right) \rightarrow 0 .
$$

Thus $\lim \sigma_{m}^{\prime}$ exists, as according to the supposition $\lim \sigma_{m}$ exists.

The convergence of $s_{n}^{\prime}$ then follows from $\left(\mathrm{H}_{1}\right)$, and because of $s_{n}-s_{n}^{\prime} \rightarrow 0$ the sequence $s_{n}$ is also convergent.

THEOREM 1*. If for the method $A$ the high indices theorem $\left(\mathrm{H}_{1}\right)$ is valid for all series satisfying the additional condition

$$
s_{n}=o(k(n)),
$$

$k=k(n)$ being defined by $n_{k} \leqq n<n_{k+1}$ and if

$$
\sum_{n_{k} \leq n<n_{k+1}} c_{n}=O(1)
$$

then $\left(\mathrm{T}_{1}\right)$ also is valid for $A$.

The proof is given in the same manner as above, the only difference being that we have to prove that $s_{n}^{\prime}=o(k(n))$ for the sequence $s_{n}^{\prime}$, in order to be able to use the theorem $\left(\mathrm{H}_{1}\right)$. But according to (3) and (5), $s_{n}=o(k(n))$, hence the same estimate holds also for $s_{n}^{\prime}$.

Similarly we can prove the following theorem.

THEOREM 2. The proposition $\left(\mathrm{T}_{2}\right)$ is a consequence of $\left(\mathrm{H}_{2}\right)$ if the $c_{n}$ satisfy the condition (5).

ThEOREM 2*. The proposition $\left(\mathrm{T}_{2}\right)$ is a consequence of $\left(\mathrm{H}_{2}\right)$ for all series 
satisfying (7) and the additional condition

$$
s_{n}=O(k(n)) .
$$

A further relation among Tauberian theorems can be obtained by means of the following theorem of Mazur and Orlicz [9] ( $\left.{ }^{1}\right)$; we do not possess any proof of this theorem. If, for a regular method $A$, no unbounded $A$-summable sequences exist, then only convergent sequences are $A$-summable.

By application of this theorem to the method $\tilde{A}$ with matrix

$$
\tilde{a}_{m k}=\sum_{n_{k} \leqq n<n_{k+1}} a_{m n}
$$

we obtain the following theorem. The high indices theorem $\left(\mathrm{H}_{1}\right)$ follows from $\left(\mathrm{H}_{2}\right)$.

In spite of their simplicity, these theorems seem to offer a certain amount of interest. By means of Theorem 1, o-Tauberian theorems can be derived with exact order of the $u_{n}$. Thus for example we deduce from the high indices theorem of Hardy and Littlewood [3], according to which $\left(\mathrm{H}_{1}\right)$ is true for the Abel (or Euler) power series method $P$ when $n_{k}=2^{k}$, the following theorem of Tauber. If the series $\sum u_{n}$ is $P$-summable and $u_{n}=o(1 / n)$, then the series is convergent.

Furthermore, by use of a theorem of Pitt [12] and Theorem $1^{*}$ we see that the estimate $u_{n}=o\left(n^{-1 / 2}\right)$ implies the convergence of $\sum u_{n}$ when $\sum u_{n}$ is Borel summable. Pitt's theorem states that if, for a $B$-summable series $\sum u_{n},(2)$ is valid with $n_{k+1}-n_{k} \geqq a\left(n_{k}\right)^{-1 / 2}, a>0$, and $s_{n}=O\left(\lambda^{n}\right)$ for every $\lambda>1$, then $\sum u_{n}$ is convergent. In fact we have only to observe that from $n_{k+1}-n_{k} \geqq a\left(n_{k}\right)^{1 / 2}, a>0$, we can easily derive $n_{k} \geqq k^{2} / q^{2}$ with a certain constant $q>0$. This means that for the function $k(n)$, defined in Theorem $1^{*}$, we have $k(n) \leqq q n^{1 / 2}$. For a result, similar to Pitt's, for the method $E_{1}$ of EulerKnopp, see Meyer-König [11].

It is scarcely possible to derive, by the same simple method, the exact $O$-Tauberian theorems containing the conditions $u_{n}=O(1 / n)$ and $O\left(1 / n^{1 / 2}\right)$ respectively. These $O$-Tauberian theorems are connected with much more delicate properties of the matrix of a transformation than those made use of in Theorem 1. See, for example, Karamata [6, p. 20].

We shall deduce from Theorem 1* that Pitt's theorem cannot in its essentials be rendered more precise. For each $\epsilon>0, a>0$, and an increasing sequence of indices $n_{k}$ with

$$
n_{k+1}-n_{k} \leqq a n_{k}^{1 / 2-\epsilon} \quad(k=1,2, \cdots)
$$

a divergent $B$-summable series exists, satisfying $s_{n}=o(n)$ and (2). For obviously $k(n) \leqq n$; If there were no such series, then Theorem $\left(\mathrm{H}_{1}\right)$ with the

(1) Numbers in brackets refer to the references cited at the end of the paper. 
additional condition $s_{n}=o(k(n))$ would be correct for the method $B$. Let $c_{n}=n^{-1 / 2+e}$, then $(7)$ is fulfilled:

$$
\sum_{n_{k} \leqq n<n_{k+1}} c_{n} \leqq n_{k}^{-1 / 2+\epsilon}\left(n_{k+1}-n_{k}+1\right)=O(1) .
$$

Then according to Theorem $1^{*}$ the condition $u_{n}=O\left(n^{-1 / 2+c}\right)$ would be a Tauberian condition, and this for the method $B$ is not the case for any $\epsilon>0$ according to Hardy and Littlewood [2, p. 15].

3. The general forms of the $O$-Tauberian theorem for the Abel and Cesàro methods. We shall say that a sequence $c_{n}, 0 \leqq c_{n} \leqq+\infty$ has the property (E) when

(E) For each $\epsilon>0$ there is a sequence of positive numbers $n_{k}$ for which $n_{k+1} / n_{k} \geqq q>1$ and

$$
\sum_{n_{k}<n<n_{k+1}} c_{n}<\epsilon .
$$

It obviously does not matter whether we suppose in this condition the $n_{k}$ to be any positive number or only integers. The supposition chosen renders the proof slightly easier. For treatments of general Tauberian conditions, see Pitt [12] and Agnew [1].

THEOREM 3. The condition (E) is the necessary and sufficient condition for the sequence $0 \leqq c_{n} \leqq+\infty$ in order that

$$
u_{n}=O\left(c_{n}\right)
$$

is a Tauberian condition for the methods $C_{\alpha}(\alpha>0)$ of Cesàro or the Abel method $P$.

Proof. (a) The condition (E) is necessary. We investigate the method $C_{1}$ and shall show first of all that, $\epsilon>0$ being given, the indices $n_{k}$ for which $c_{n_{k}} \geqq \epsilon$ constitute a sequence with $n_{k+1} / n_{k} \geqq q>1$, if there is an infinite number of them. Otherwise we should have an $\epsilon_{0}>0$ and two sequences of integers $m_{k}$, $l_{k}$ with $m_{k}<l_{k}<m_{k+1}, l_{k} / m_{k} \rightarrow 1, c_{m_{k}} \geqq \epsilon_{0}, c_{l_{k}} \geqq \epsilon_{0}$. By considering a partial sequence we can regard the following as fulfilled:

$$
\left[\left(l_{1}-m_{1}\right)+\left(l_{2}-m_{2}\right)+\cdots+\left(l_{k}-m_{k}\right)\right] / m_{k} \rightarrow 0 .
$$

Under these conditions, let

$$
\begin{array}{rlrl}
u_{n} & =+\epsilon_{0} & \left(n=m_{k}, k=1,2, \cdots\right) \\
& =-\epsilon_{0} & & \left(n=l_{k}, k=1,2, \cdots\right) \\
& =0 & & \text { (for all remainlng } n) .
\end{array}
$$

Then for the $C_{1}$ transform $\sigma_{n}$ of the series $\sum u_{n}$ we have, when $m_{k} \leqq n<m_{k+1}$,

$$
0 \leqq \sigma_{n} \leqq\left[\left(l_{1}-m_{1}\right)+\cdots+\left(l_{k}-m_{k}\right)\right] \epsilon_{0} / m_{k} \rightarrow 0 .
$$


Furthermore $\left|u_{n}\right| \leqq c_{n}$. As the series $\sum u_{n}$ diverges, (9) would not be a Tauberian condition for the method $C_{1}$.

We shall now prove, again for the method $C_{1}$, that the sequence $c_{n}$ has the property (E). Suppose (E) is not fulfilled. Then an $\epsilon_{0}>0$ exists for which there is no sequence of positive numbers $n_{k}$ which fulfills the requirements of (E). Thus for every sequence $n_{k}$, with $n_{k+1} / n_{k} \geqq q>1, \sum_{n_{k}<n<n_{k+1}} c_{n} \geqq \epsilon_{0}$ holds even for an infinity of $k$.

Let $n_{\mathbf{k}}^{0}$ be a sequence as mentioned above, for which $c_{n}<\epsilon_{0} / 3$ for $n \neq n_{k}^{0}$. We can suppose

$$
1<q_{0} \leqq n_{k+1}^{0} / n_{k}^{0} \leqq Q_{0}<+\infty \quad(k=1,2, \cdots) .
$$

From the sequence $n_{k}^{0}$ we form the sequence $n_{k}^{1}$ whose elements are

$$
\stackrel{0}{n_{1}},\left(\stackrel{n_{1}^{0} n_{2}^{0}}{0}\right)^{1 / 2}, \stackrel{n_{2}^{0}}{0},\left(n_{2}^{0} n_{3}^{0}\right)^{1 / 2}, n_{3}^{0}, \cdots .
$$

Similarly from $n_{k}^{1}$ we form the sequence $n_{k}^{2}$, and so on. Then for each sequence $n_{k}^{p}$ we evidently have

$$
1<q_{p} \leqq n_{k+1}^{p} / n_{k}^{p} \leqq Q<\infty \quad(k=1,2, \cdots)
$$

where

$$
q_{p}=\left(q_{0}\right)^{1 / 2^{p}}, \quad Q_{p}=\left(Q_{0}\right)^{1 / 2^{p}} .
$$

According to the above, we can find numbers

$$
N_{s}=n_{k_{s}}^{p_{s}}, \quad N_{s}^{\prime}=n_{k_{s}+1}^{p_{s}}, \quad(s=1,2, \cdots)
$$

such that

$$
N_{s} \rightarrow \infty, \quad p_{s} \rightarrow \infty, \quad N_{s}<N_{s}^{\prime}<N_{s+1}, \quad \sum_{N_{s}<n<N_{s}} c_{n} \geqq \epsilon_{0} .
$$

Since, according to (10), as $s \rightarrow \infty$,

$$
0 \leqq \frac{1}{N_{s}}\left(N_{s}^{\prime}-N_{s}\right)=\frac{N_{s}^{\prime}}{N_{s}}-1 \leqq Q_{p_{s}}-1 \rightarrow 0
$$

we can even achieve

$$
\left[\left(N_{1}^{\prime}-N_{1}\right)+\left(N_{2}^{\prime}-N_{2}\right)+\cdots+\left(N_{s}^{\prime}-N_{s}\right)\right] / N_{s} \rightarrow 0
$$

by taking a partial sequence of the $s$.

As $c_{n}<\epsilon_{0 / 3}$ for $N_{s}<n<N_{s}^{\prime}$ and on account of the last inequality (11) there is an $\bar{N}_{8}$ between $N_{s}$ and $N_{\varepsilon}^{\prime}$ such that

$$
\sum_{N_{s}<n \geqq \bar{N}_{s}} c_{n} \geqq \epsilon_{0} / 3, \quad \sum_{N_{s}<n<N_{\bullet}} c_{n} \geqq \epsilon_{0} / 3 .
$$

(One has merely to choose a first $\bar{N}_{\mathrm{g}}$ for which the first of these inequalities is fulfilled.) 
We now define $u_{n}$ to be positive in $N_{8}<n \leqq \bar{N}_{8}$ and negative in $\bar{N}_{8}<n<N_{8}^{\prime}$ such that $\left|u_{n}\right| \leqq c_{n}$ and

$$
\sum_{N_{\iota}<n \leqq \bar{N}_{\bullet}} u_{n}=\epsilon_{0} / 3, \quad \sum_{\bar{N}_{\iota}<n<N_{\bullet}^{\prime}} c_{n}=-\epsilon_{0} / 3 .
$$

For the remaining $n$ let $u_{n}=0$. Then $\sum u_{n}$ is divergent and $\left|u_{n}\right| \leqq c_{n}$ holds for all $n$, but $\sigma_{m}$ converges toward zero. For we have $s_{n}=0$ for any $n$ outside the intervals $N_{8}<n<N_{s}^{\prime}$ and $0 \leqq s_{n} \leqq \epsilon_{0} / 3$ within these intervals. Thus for $N_{s} \leqq n<N_{s+1}$ we have

$$
0 \leqq \sigma_{n} \leqq\left[\left(N_{1}^{\prime}-N_{1}\right)+\left(N_{2}^{\prime}-N_{2}\right)+\cdots+\left(N_{s}^{\prime}-N_{s}\right)\right] \epsilon_{0} / 3 N_{s} \rightarrow 0 .
$$

Hence (9) is not a Tauberian condition for the method $C_{1}$. We thus have a contradiction.

Regarding the methods $C_{\alpha}(\alpha>0)$ and $P$, the necessity of (E) follows for them from the fact that they contain the method $C_{1}$. Finally according to a theorem by Andersen the methods $C_{\alpha}(\alpha>0)$ and $C_{1}$ are equivalent for series with bounded partial sums; see, for example, Zygmund [14, p. 262]. Hence the above proof, making use of series of this kind only, remains valid for $C_{\alpha}, 0<\alpha<1$, as well as for $C_{1}$.

(b) We shall now show that (E) is sufficient. This part of Theorem 3 is not new. It follows from a theorem of Pitt [12, Theorem 13]. Pitt considers more general methods, and his proof is much more complicated than the one given here. A proof of Agnew [1, Theorem 9.21] of a $C_{1}$ Tauberian theorem, with a very general Taublerian condition, is more like the proof given below. Suppose (E) and (9) to be fulfilled, and let $s_{n}$ be $P$-summable. Since Ingham [4] has shown that the high indices theorem $\left(H_{2}\right)$ for the Abel method is valid for any sequence $\left\{n_{k}\right\}$ with $n_{k+1} / n_{k} \geqq q>1$, we see in accordance with Theorem 2 that the sequence $s_{n}$ is bounded. As the methods $P$ and $C_{1}$ are equivalent for such sequences (see, for example, Landau [7, p. 12]), we first obtain the $C_{1}$-summability of the sequence $s_{n}$. Its convergence follows in the known manner:

Without restriction of generality, we can suppose $\left|u_{n}\right| \leqq c_{n}$ and $\sigma_{n}=\left(s_{1}+\cdots+s_{n}\right) / n \rightarrow 0$. We choose any $\epsilon>0$ and a sequence $n_{k}$ in accordance with (E). For all $k_{0}$ sufficiently large we have $\left|\sigma_{n}\right|<\epsilon$ for $n \geqq n_{k_{0}}-1$. Hence from

$$
\sigma_{n_{k+1}-1}=\frac{s_{1}+\cdots+s_{n_{k}-1}}{n_{k+1}-1}+\frac{s_{n_{k}}+\cdots+s_{n_{k+1}-1}}{n_{k+1}-1}
$$

for $k \geqq k_{0}, n_{k} \leqq n<n_{k+1}$ we have, on account of $\left|s_{m}-s_{n}\right|<\epsilon$ for $n_{k} \leqq m<n_{k+1}$, that

$$
\sigma_{n_{k+1}-1}=\frac{n_{k}-1}{n_{k+1}-1} \sigma_{n_{k}-1}+\frac{n_{k+1}-n_{k}}{n_{k+1}-1} s_{n}+\theta \frac{n_{k+1}-n_{k}}{n_{k+1}-1} \epsilon
$$


where $|\theta|<1$. Thence we deduce for the $s_{n}$

$$
\left|s_{n}\right| \leqq \epsilon+\frac{2 n_{k+1}}{n_{k+1}-n_{k}} \epsilon \leqq\left(1+\frac{2 q}{q-1}\right) \epsilon,
$$

that is, $s_{n} \rightarrow 0$. This completes the proof of Theorem 3 .

An analogous theorem can be proved for the one-sided Tauberian condition. Instead of condition (E), we now introduce condition $(F)$ for the sequence $0 \leqq c_{n} \leqq+\infty$ which shall signify:

(F) For each $\epsilon>0$, there is a sequence $n_{k}$ of positive numbers with $n_{k+1} / n_{k} \geqq q>1$ for which

$$
\sum_{n_{k} \leqq n<n_{k+1}} c_{n}<\epsilon .
$$

Thus while in (E) the possibility $c_{n}=+\infty$ for an infinity of $n$ was not excluded, nearly all $c_{n}$ are finite in this case. The theorem mentioned can be stated as follows:

THEOREM 4. The condition $(\mathrm{F})$ is the necessary and sufficient condition for the sequence $0 \leqq c_{n} \leqq+\infty$ in order that

$$
u_{n} \leqq O\left(c_{n}\right)
$$

is a Tauberian conditions for the methods $C_{\alpha}(\alpha>0)$ of Cesaro or the Abel method $P$.

Proof. (a) The condition (F) is necessary. We shall first prove that $c_{n} \rightarrow 0$. Otherwise there would be a sequence of indices $l_{k} \rightarrow \infty$ with $c_{l_{k}} \geqq \epsilon_{0}>0$. We may suppose $l_{k+1}>l_{k}+1$ and furthermore

$$
k / l_{k} \rightarrow 0 \quad \text { for } k \rightarrow \infty .
$$

Let $u_{n}=-1$ when $n=l_{k}-1, k=2,3, \cdots ; u_{n}=+1$ when $n=l_{k}, k=2,3, \cdots$; and $u_{n}=0$ for all remaining $n$. We have $u_{n} \leqq O\left(c_{n}\right)$ and the respective $s_{n}$ constitute a bounded sequence. This sequence is $C_{1}$-summable on account of (13), hence also $C_{\alpha}(\alpha>0)$ and $A$-summable. But it is divergent and therefore (12) is not a Tauberian condition.

Now according to Theorem 3 the condition (E) must be fulfilled. This together with $c_{n} \rightarrow 0$ gives $(\mathrm{F})$, if we omit some of the first $n_{k}$ if necessary.

(b) The sufficiency of (F) can be deduced from a theorem by R. Schmidt $[13$, Theorem 11] according to which

$$
\limsup _{\delta \rightarrow 0} \phi(\delta) \leqq 0 ; \quad \phi(\delta)=\limsup _{m \rightarrow \infty} \max _{m \leqq 0 \leqq w \leqq m(1+\delta)}\left(s_{w}-s_{v}\right)
$$

represents a Tauberian condition for the Abel method.

Suppose $(F)$ to be fulfilled. We then choose the sequence $n_{k}$ for a given $\epsilon>0$ in accordance with (F). If then $\delta>0$ is so small that $1+\delta<q$ and if 
$n_{k} \leqq m<n_{k+1}$, then surely $m(1+\delta)<n_{k+2}$ and therefore

$$
s_{w}-s_{v}=u_{v+1}+\cdots+u_{w} \leqq M \sum_{n_{k} \leqq n<n_{k+2}} c_{n}<2 M \epsilon
$$

with a constant $M>0$ for $m \leqq v \leqq w \leqq m(1+\delta)$. Thus $\phi(\delta) \leqq 2 M \epsilon$ and (14) is fulfilled. This completes the proof of Theorem 4 .

Now we shall add some consequences to illustrate the applicability of the theorems proved above.

1. Let $\omega(n) \rightarrow \infty$ for $n \rightarrow \infty$. Then $u_{n}=O(\omega(n) / n)$ is not a Tauberian condition for the methods $C_{\alpha}(\alpha>0)$ and $P$. (For the Abel method, this was proved by Littlewood [8].) For the condition (E) is not fulfilled here:

$$
\begin{aligned}
\sum_{n_{k}<n<n_{k+1}} c_{n} & =\sum_{n_{k}<n<n_{k+1}} \omega(n) / n \geqq \omega\left(n_{k}\right)\left(n_{k+1}-n_{k}-2\right) / n_{k+1} \\
& \geqq \omega\left(n_{k}\right)\left(1-q-\frac{2}{n_{k+1}}\right) \rightarrow \infty .
\end{aligned}
$$

2. If $c_{n}$ is not increasing, $u_{n}=O\left(c_{n}\right)$ or also $u_{n} \leqq O\left(c_{n}\right)$ is then and then only a Tauberian condition, when for every $\epsilon>0$ there is a $\delta>0$ such that for all $n$ sufficiently large

$$
\sum_{n<v<m(1+\delta)} c_{v}<\epsilon
$$

For (F) follows from this property of the $c_{n}$ if we choose $n_{k}=(1+\delta)^{k}$ and add sufficiently many of the first integers to these $n_{k}$. Conversely, (15) follows from (F) or (E) as can easily be proved on the same lines as (b), Theorem 4.

3. Menchoff [10] published a theorem which implies that $u_{n}=O\left(c_{n}\right)$ with

$$
\sum_{n=2^{k}}^{n=2 k+1} c_{n}=O(1)
$$

is a Tauberian condition for the method $P$. This theorem was later withdrawn by him [10]. Now it is easy to form a sequence $c_{n}$ for which (16) holds although (E) is not fulfilled. (For instance, let $c_{2^{k}}=c_{2^{k}+1}=1$, and $c_{n}=0$ for other $n$.) Hence (16) is indeed not a Tauberian condition.

4. If $n_{k}$ is a sequence with $n_{k+1} / n_{k} \geqq q>1$ and if for a series $\sum u_{n}$ which is $P$-summable, $u_{n}=O(1 / n)$ holds for $n \neq n_{1}, n_{2}, \cdots$, then the series is convergent. (For the Cesàro methods $C_{\alpha}$, see Meyer-König [11].)

Let $c_{n}=M / n$ for $n \neq n_{k}, c_{n}=+\infty$ for $n=n_{1}, n_{2}, \cdots$. For a given $\epsilon>0$ we then choose the sequence $m_{k}$ such that it contains all the $n_{k}$ and that $1<q^{\prime} \leqq m_{k+1} / m_{k}<1+\epsilon$. Then for every $k$ we have

$$
\sum_{m_{k}<n<m_{k+1}} c_{n} \leqq \frac{M}{m_{k}}\left(m_{k+1}-m_{k}\right)<M \epsilon,
$$


that is, the condition (E) is fulfilled.

5. Suppose $M_{n} \geqq 0$ to be a $C_{1}$-summable sequence. Then

$$
u_{n} \leqq M_{n} / n
$$

is a Tauberian condition for the methods $C_{\alpha}$ and $P$. (See Karamata [5].)

We have only to prove that the numbers $c_{n}=M_{n} / n$ satisfy the condition (F). For a given $\epsilon>0$ let $n_{k}=(1+\epsilon)^{k}$. We shall designate $\sigma_{k}=\left(1 / n_{k}\right) \sum_{n<n_{k}} M_{n}$ and $\sigma=\lim \sigma_{k}$. Then

$$
\begin{aligned}
\sum_{n_{k} \leqq n<n_{k+}} c_{n} & \leqq \frac{1}{n_{k}} \sum_{n_{k} \leqq n<n_{k+1}} M_{n}=\frac{1}{n_{k}}\left(n_{k+1} \sigma_{k+1}-n_{k} \sigma_{k}\right) \\
& =\epsilon \sigma_{k+1}+\left(\sigma_{k+1}-\sigma_{k}\right) .
\end{aligned}
$$

For $k$ sufficiently large $\left|\sigma_{k+1}-\sigma_{k}\right|<\epsilon, \sigma_{k+1}<\sigma+1$ and therefore the sum to be estimated is less than

$$
\epsilon(\sigma+1)+\epsilon=\epsilon(\sigma+2) .
$$

\section{REFERENCES}

1. R. P. Agnew, Tauberian conditions, Ann. of Math. vol. 42 (1941) pp. 293-308.

2. G. H. Hardy and J. E. Littlewood, The relations between Borel's and Cesàro's methods of summation, Proc. London Math. Soc. vol. 11 (1913) pp. 1-16.

3. - A further note on the converse of Abel's theorem, Proc. London Math. Soc. vol. 25 (1926) pp. 219-236.

4. A. E. Ingham, On the "high-indices theorem" of Hardy and Littlewood. Quart. J. Math. Oxford Ser. vol. 8 (1937) pp. 1-7.

5. J. Karamata, Théorèmes inverses de sommabilité I, II, Glas Srpske Kralevske Akad., Beograd, vol. 143 (70) (1931) pp. 3-24, 121-146.

6. - Sur les théorèmes inverses des procédés de sommabilité, Paris, 1937, $46 \mathrm{pp}$.

7. E. Landau, Neuere Ergebnisse der Funktionentheorie, 2d ed., Berlin, 1929, 122 pp.

8. J. E. Littlewood, The converse of Abel's theorem on power series, Proc. London Math. Soc. vol. 9 (1911) pp. 434-443.

9. S. Mazur and W. Orlicz, Sur les méthodes linéaires de sommation, C. R. Acad. Sci. Paris vol. 196 (1933) pp. 32-34.

10. D. Menchoff, Sur une généralisation d'une theorème de M. M. Hardy et Littlewood, Rec. Math. (Mat. Sbornik) N.S. vol. 3 (1938) pp. 367-373; vol. 5 (1939) p. 451.

11. W. Meyer-König, Limitierungsumkehrsätze mit Lïckenbedingungen I; II, Math. Zeit. vol. 45 (1939) pp. 447-478; 479-494.

12. H. R. Pitt, General Tauberian theorems, Proc. London Math. Soc. vol. 44 (1938) pp. 243-288.

13. R. Schmidt, Über divergenten Folgen und lineare Mittelbildungen, Math. Zeit. vol. 22 (1925) pp. 89-152.

14. A. Zygmund, Trigonometrical series, Warsaw-Lw6w, $1935331 \mathrm{pp}$.

FRANKFURT, GERMANY. 